\title{
Apolipoprotein(a) isoform-specific changes of lipoprotein(a) after kidney transplantation
}

\author{
Florian Kronenberg ${ }^{*}, 1,2$, Karl Lhotta ${ }^{3}$, Paul König ${ }^{3}$, Raimund Margreiter ${ }^{4}$, Hans Dieplinger ${ }^{1}$ \\ and Gerd Utermann ${ }^{1}$ \\ ${ }^{1}$ Institute of Medical Biology and Human Genetics, University of Innsbruck, Austria; ${ }^{2}$ Institute of Epidemiology, \\ GSF-National Research Center for Environment and Health, Neuherberg, Germany; ${ }^{3}$ Department of Clinical Nephrology, \\ Innsbruck University Hospital, Austria; ${ }^{4}$ Department of Transplant Surgery, Innsbruck University Hospital, Austria
}

The atherogenic lipoprotein(a) $(\operatorname{Lp}(a))$ is significantly increased in patients with kidney disease. Some studies in hemodialysis patients described this increase to be dependent on the genetic apolipoprotein(a) (apo(a)) isoforms. Only patients who express high molecular weight (HMW) apo(a) isoforms but not those with low molecular weight (LMW) isoforms show a relative increase of $L p(a)$ when compared to healthy controls matched for apo(a) isoforms. However, this was not confirmed by all studies. We therefore prospectively investigated the changes of $\operatorname{Lp}(a)$ deriving from each apo(a) isoform in heterozygotes following kidney transplantation. $\operatorname{Lp}(a)$ concentrations were measured by ELISA. To calculate the isoformspecific concentrations and the changes of $\operatorname{Lp}(a)$ deriving from each isoform, we densitometrically scanned the apo(a) bands from immunoblots before and after transplantation in 20 patients expressing two apo(a) isoforms. Of these, 10 patients expressed both an LMW and an HMW apo(a) isoform. The other 10 patients expressed only HMW isoforms. Densitometric scanning of apo(a) bands and calculation of isoform-derived $L p(a)$ concentrations clearly demonstrated that the decrease of $L p(a)$ following kidney transplantation is caused by changes in the expression of HMW apo(a) isoforms. In some patients, we observed an almost complete disappearance of the HMW apo(a) isoform after transplantation. This study clearly demonstrates that the changes of $L p(a)$ plasma concentrations in kidney disease depend on the genetically determined size of apo(a). This provides evidence for an interaction of apo(a) genetic variability and kidney function on $\operatorname{Lp}(\mathrm{a})$ concentrations.

European Journal of Human Genetics (2003) 11, 693-699. doi:10.1038/sj.ejhg.5201016

Keywords: lipoprotein(a); apo(a) isoforms; kidney transplantation; kidney disease; interaction; gene-gene interaction; gene-environment interaction

\section{Introduction}

Lipoprotein(a) $(\operatorname{Lp}(a))$ is the lipoprotein with the strongest genetic determination of its plasma concentrations. Some $70-90 \%$ of the variation in $\mathrm{Lp}(\mathrm{a})$ concentrations are determined by sequence variations at the apolipoprotein(a) (apo(a)) gene locus. The kringle-IV (K-IV) repeat poly-

*Correspondence: Dr F Kronenberg, Institute of Medical Biology and Human Genetics, Schöpfstr. 41, A-6020 Innsbruck, Austria. Tel: +43 512 507 3474; Fax: +43 5125072680 or - 2861;

E-mail: Florian.Kronenberg@uibk.ac.at

Received 8 January 2003; revised 16 March 2003; accepted 21 March 2003 morphism accounts for a major part of this variation. ${ }^{1-4}$ However, some pathological conditions are known to influence $\operatorname{Lp}(\mathrm{a})$ plasma concentrations. ${ }^{5}$ One of the most important secondary causes of an $\mathrm{Lp}$ (a) elevation is kidney disease (reviewed in Kronenberg et $a l^{6}$ ).

We described in two earlier studies an apo(a) phenotypedependent Lp(a) elevation in hemodialysis patients. Only patients with high molecular weight (HMW) apo(a) phenotypes had on average elevated Lp(a) plasma concentrations when compared to apo(a) phenotype-matched controls. Those with low molecular weight (LMW) apo(a) phenotypes showed similar $\operatorname{Lp}(\mathrm{a})$ concentrations than 
matched controls. ${ }^{7,8}$ Although these findings were recently supported by two studies, ${ }^{9,10}$ three other reports did not confirm our observation and described an elevation of Lp(a) in all apo(a) phenotype groups. ${ }^{11-13}$ Since our original observation suggested a rare example of a disease influence on the allele-specific expression of a protein in humans, we have reinvestigated the problem using a more rigorous approach.

We demonstrate in this investigation a pronounced change in the relative expression of the higher in comparison to the lower molecular weight apo(a) isoform following successful kidney transplantation in heterozygous patients with two expressed apo(a) alleles in plasma. By densitometrical scanning of the apo(a) bands, we were able for the first time to calculate the isoform-specific $\mathrm{Lp}(\mathrm{a})$ concentration changes caused by restoration of kidney function. This strongly supports the finding that the elevation of $\operatorname{Lp}(\mathrm{a})$ in hemodialysis patients is apo(a) genotype-related. ${ }^{7,8}$

\section{Material and methods Patients}

We screened a group of 57 hemodialysis patients from the participating renal unit for the presence of two clearly visible apo(a) bands before transplantation with a difference of at least five K-IV repeats. Of them, 20 fulfilled these criteria and are the basis of the present study. The 11 women and nine men were on average $44 \pm 10$ years old. The primary kidney disease was chronic glomerulonephritis in 12 patients, polycystic kidney disease in three patients, diabetic nephropathy in two patients, chronic pyelonephritis in one patient, and unknown reasons in two cases. The preoperative as well as the postoperative drug treatment including the immunosuppressive therapy are described in detail elsewhere. ${ }^{14}$

A baseline plasma sample was obtained immediately before kidney transplantation from all patients. In 13 patients two further samples were withdrawn, one on average 3 weeks (short follow-up) and one on average 57 months following transplantation (long follow-up). In seven patients, we could not obtain plasma samples from the long follow-up. Plasma samples were frozen and kept at $-80^{\circ} \mathrm{C}$ prior to analysis. ${ }^{15}$

Hematocrit has a strong influence on Lp(a) levels when measured in plasma. ${ }^{16}$ This is especially relevant in longitudinal studies with potential changes of hematocrit as it is usually the case after kidney transplantation. We therefore corrected $\mathrm{Lp}(\mathrm{a})$ plasma concentrations measured after kidney transplantation for the observed hematocrit changes by the following formula:

$$
\begin{aligned}
& \text { corrected } \mathrm{Lp}(\mathrm{a}) \text { level } \\
& =\text { measured } \mathrm{Lp}(\mathrm{a}) \text { level } \frac{1-\text { hematocrit after transplantation }}{1-\text { hematocrit before transplantation }}
\end{aligned}
$$

This resulted on average in a correction of $+8.7 \%$ for the measured $\mathrm{Lp}(\mathrm{a})$ levels after the short-term followup and $-5.8 \%$ after the long-term follow-up.

\section{Laboratory procedures}

Lp(a) quantification was performed as described elsewhere $^{15}$ with a double-antibody ELISA using an affinity purified polyclonal apo(a) antibody for coating and the horseradish peroxidase-conjugated monoclonal $1 \mathrm{~A} 2$ for detection. Apo(a) phenotyping was performed by sodium dodecyl sulfate-agarose gel electrophoresis (SDS agarose) ${ }^{17}$ under reducing conditions, as previously outlined, ${ }^{18}$ with the following modifications: we applied from each sample the same amount of apo(a) to the gel. The dilution factor of the plasma sample in the sample buffer (described in Kraft et $a l^{18}$ ) was obtained by the measured $\mathrm{Lp}(\mathrm{a})$ concentration times 0.8 . We used $10 \times 2 \mathrm{~mm}^{2}$ combs to apply $20 \mu \mathrm{l}$ of the prepared sample to the gel. This corresponds to $250 \mathrm{ng}$ of Lp(a). Electrophoresis was followed by immunoblotting with the same monoclonal antibody $1 \mathrm{~A} 2$ for detection of apo(a) isoforms as used in the ELISA. ${ }^{19}$

After immunoblotting we scanned apo(a) bands by a Personal Densitometer SI (Molecular Dynamics, Sunnyvale, USA) and compared the relation of the lower to the higher molecular weight apo(a) isoforms before and following transplantation. We used the $\mathrm{Lp}(\mathrm{a})$ concentrations measured by ELISA to calculate the isoform-specific amount of $\mathrm{Lp}$ (a) derived from each isoform by using these densitometric relative estimates as recently described. ${ }^{20}$ For example, when an individual with 21 and $33 \mathrm{~K}-\mathrm{IV}$ repeats had an Lp(a) serum concentration of $40 \mathrm{mg} / \mathrm{dl}$ measured by ELISA, and the $21 \mathrm{~K}-\mathrm{IV}$ isoform accounted for $85 \%$ of the staining in the SDS agarose gel electrophoresis, we calculated the $\operatorname{Lp}(\mathrm{a})$ concentration originating from this isoform to be $34 \mathrm{mg} / \mathrm{dl}(40 \times 0.85)$. The isoform with $33 \mathrm{~K}$ IV repeats accounted accordingly for the remaining $6 \mathrm{mg} /$ dl. By this procedure, we were able to calculate the apo(a) isoform-associated changes of $\mathrm{Lp}(\mathrm{a})$ concentrations.

The term LMW apo(a) phenotype was used when a subject expressed at least one apo(a) isoform with 11-22 K-IV repeats. ${ }^{18}$ A patient was considered to have an HMW apo(a) phenotype when expressing only isoforms of high molecular weight ( $>22 \mathrm{~K}-\mathrm{IV}$ repeats). ${ }^{21}$

\section{Statistical procedures}

The $\operatorname{Lp}(\mathrm{a})$ concentrations as well as the relative changes of $\mathrm{Lp}$ (a) before and after kidney transplantation were compared by the paired Wilcoxon rest.

\section{Results}

In all, 10 patients showed an LMW apo(a) phenotype meaning that they expressed one LMW apo(a) isoform ( $\leqslant 22$ K-IV repeats) and one HMW apo(a) isoform. The mean Lp(a) concentration was $42.3 \pm 33.8 \mathrm{mg} / \mathrm{dl}$ before transplantation, which decreased to $33.0 \pm 29.8 \mathrm{mg} / \mathrm{dl} 3$ weeks after transplantation $(P=0.11)$. We densitometrically scanned the expressed isoforms and calculated the isoform-associated $\mathrm{Lp}(\mathrm{a})$ concentrations for each allele. 
This revealed that $L p(a)$ deriving from the LMW apo(a) isoform remained almost unchanged after transplantation $(29.0 \pm 30.1$ vs $30.1 \pm 29.0 \mathrm{mg} / \mathrm{dl}, P=0.65)$ (Table 1$)$. Virtually all of the observed decrease was caused by changes in the expression of the HMW apo(a) isoform: $\mathrm{Lp}(\mathrm{a})$ deriving from these isoforms decreased from $13.3 \pm 13.5$ before transplantation to $2.9 \pm 3.7 \mathrm{mg} / \mathrm{dl}$ after transplantation $(P=0.013)$.

The remaining 10 patients expressed both isoforms as HMW apo(a) isoforms. Lp(a) decreased in these patients from $31.7 \pm 33.6 \mathrm{mg} / \mathrm{dl}$ before transplantation to $21.5 \pm 27.1 \mathrm{mg} / \mathrm{dl}$ after transplantation $(P=0.007)$. When we grouped the smaller of the two HMW isoforms from each patient together in one isoform group and the larger ones in a second isoform group, we found that both HMW isoform groups contributed to this decrease. $\mathrm{Lp}$ (a) levels of the group of the smaller HMW apo(a) isoform decreased from $23.0 \pm 29.4$ to $16.5 \pm 21.7 \mathrm{mg} / \mathrm{dl}(P=0.037)$. Those from the larger HMW isoform group decreased from $8.7 \pm 6.2$ to $5.0 \pm 6.8 \mathrm{mg} / \mathrm{dl}(P=0.059)$.

The differences in the changes of $\operatorname{Lp}(\mathrm{a})$ between LMW and HMW apo(a) isoform groups were not caused by differences in the transplant function. After the short-term observation period, both groups showed a decrease in creatinine to 1.51 and $1.84 \mathrm{mg} / \mathrm{dl}$ on average, respectively (Table 1).

The scatterplot in Figure 1 demonstrates a negative correlation between the number of K-IV repeats and the relative changes of apo(a) isoform-associated $\operatorname{Lp}(\mathrm{a})$ concentrations after the short follow-up period $(r=-0.65$, $P<0.001)$. Two of the three patients with LMW apo(a) isoforms that showed a relative increase in the expression of more than $50 \%$ showed a relatively low absolute $\mathrm{Lp}(\mathrm{a})$ concentration (2.76 and $6.83 \mathrm{mg} / \mathrm{dl}$, respectively).

Figure 2 shows the relative apo(a) isoform-specific changes of $\operatorname{Lp}(\mathrm{a})$ stratified for the first and second apo(a) isoform and whether these isoforms derived from patients with LMW and HMW apo(a) phenotype. It furthermore provides the long-term changes of $\mathrm{Lp}(\mathrm{a})$ concentrations in those patients who were followed for a mean period of 57 months after transplantation. A tendency for an increase in Lp(a) levels from 3 weeks to 57 months after transplantation can be seen. This effect is most pronounced for the larger

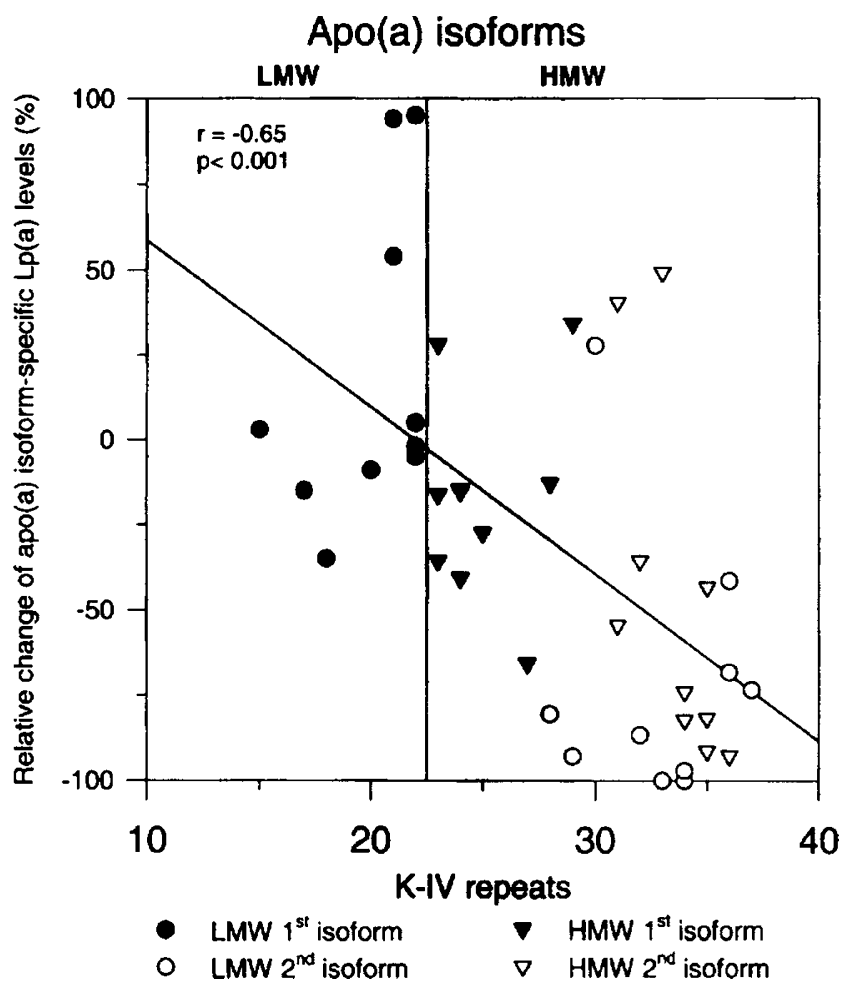

Figure 1 Scatterplot demonstrating a negative correlation between the number of K-IV repeats of apo(a) isoforms and the relative changes of $a p o(a)$ isoform-specific $L p(a)$ concentrations after the short post-transplantational follow-up period. The different symbols represent the various apo(a) isoforms: for LMW apo(a) phenotypes, closed circles represent the first apo(a) isoform (which is of LMW) and open circles represent the second isoform (which is of HMW). For HMW apo(a) phenotypes, the first isoform is shown as closed triangle, the second one is shown as open triangle (both are of $\mathrm{HMW}$ ). The vertical line represents the cutoff between apo(a) isoforms of LMW and HMW apo(a) isoforms.

Table 1 Concentrations of $\operatorname{Lp}(a)$, apo(a) isoform-specific $L p(a)$ and creatinine before and after kidney transplantation (short-term follow-up)

\begin{tabular}{|c|c|c|c|}
\hline & Before transplantation & After transplantation & $P$ \\
\hline \multicolumn{4}{|l|}{ LMW apo(a) phenotypes $(n=10)$} \\
\hline Total concentration & $42.3 \pm 33.8$ & $33.0 \pm 29.8$ & 0.11 \\
\hline Concentration of first isoform (LMW) & $29.0 \pm 30.7$ & $30.0 \pm 29.0$ & 0.65 \\
\hline Concentration of second isoform (HMW) & $13.3 \pm 13.5$ & $2.9 \pm 3.7$ & 0.013 \\
\hline Creatinine $(\mathrm{mg} / \mathrm{dl})$ & $8.19 \pm 1.92$ & $1.51 \pm 0.62$ & $<0.001$ \\
\hline \multicolumn{4}{|l|}{ HMW apo(a) phenotypes $(n=10)$} \\
\hline Total concentration & $31.7 \pm 33.6$ & $21.5 \pm 27.1$ & 0.007 \\
\hline Concentration of first isoform (HMW) & $23.0 \pm 29.4$ & $16.5 \pm 21.7$ & 0.037 \\
\hline Concentration of second isoform (HMW) & $8.7 \pm 6.2$ & $5.0 \pm 6.8$ & 0.059 \\
\hline Creatinine $(\mathrm{mg} / \mathrm{dl})$ & $9.73 \pm 2.99$ & $1.84 \pm 0.72$ & $<0.001$ \\
\hline
\end{tabular}




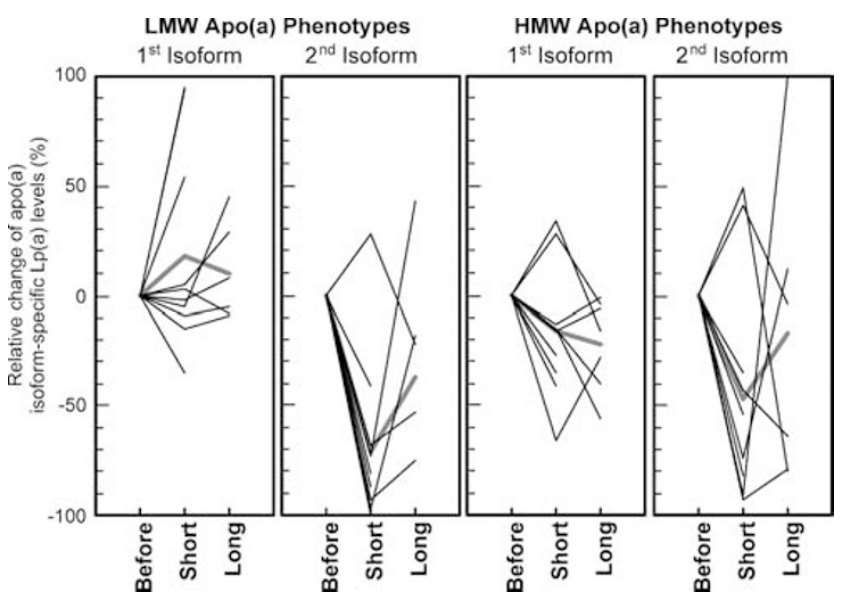

Figure 2 Individual relative changes of apo(a) isoformspecific $L p(a)$ concentrations after a short-term (on average three weeks) and a long-term (on average 57 months) observation period following kidney transplantation. Patient groups are stratified in LMW and HMW apo(a) phenotypes. 'Before' denotes the concentration before kidney transplantation. First isoform denotes the isoform with the lower number of K-IV repeats and second isoform the other isoform. The first isoform was of LMW in all 10 patients with LMW apo(a) phenotypes. The second isoform was always of HMW. The gray lines give the average changes of $L p(a)$ for the respective isoform groups.

apo(a) isoform, which was always of HMW in this patient group. Almost all of the patients with a long-term observation period, who showed an marked re-increase of the isoform-associated Lp(a) levels, had a pronounced decrease of kidney function. In long-term followed patients, the average serum creatinine concentration reincreased to 3.03 and $2.61 \mathrm{mg} / \mathrm{dl}$ for those with LMW and HMW apo(a) phenotypes, respectively.

Figure 3 shows the immunoblots of plasma apo(a) isoforms before and after transplantation from eight representative patients. The $\mathrm{Lp}(\mathrm{a})$ concentrations derived from each isoform were calculated from the percentual relation of the two apo(a) bands. These immunoblots demonstrate a strong decrease of the higher molecular weight apo(a) isoform after the short observation period (on average 3 weeks after transplantation). In some patients, the higher molecular weight apo(a) isoform almost completely disappeared (patients A, B, F, G and $\mathrm{H})$. The long-term observation period showed a reenforcement of the higher molecular apo(a) band in case of a mild or moderate re-impairment of kidney function.

\section{Discussion}

We here provide clear evidence that the effect of kidney disease on the plasma concentrations of $\operatorname{Lp}(\mathrm{a})$ depends on the apo(a) genotype. Hence, we demonstrate an interaction of the kidney function (or kidney disease) with the apo(a) gene on $\operatorname{Lp}(\mathrm{a})$ concentrations.
It is long known that the apo(a) genotype and in particular the number of K-IV repeats in the apo(a) gene have a causal effect on Lp(a) plasma concentrations. ${ }^{1-4}$ There are few nongenetic influences on $\mathrm{Lp}(\mathrm{a})$ concentrations, ${ }^{5}$ one of which is kidney disease. ${ }^{6}$ We demonstrate in the present study that the effect of kidney disease and apo(a) genotype on $\mathrm{Lp}(\mathrm{a})$ plasma concentrations is not additive but multiplicative and hence fulfills the criteria for an interaction, which may be called a disease-gene interaction (Figure 4). Since the disease under study (endstage kidney disease) may have primary environmental (eg toxic) or genetic (eg polycystic kidney disease) causes, we conclude that a gene-environmental interaction or a gene-gene interaction may underlie the observed interaction with the kidney disease. Both types of interaction manifest themselves through the same unknown mechanisms that are triggered by the kidney function regardless of the etiology of the renal insufficiency. Restoring kidney function by kidney transplantation makes this interaction clearly apparent (Figure 4).

Several observations argue for a role of the kidney in the metabolism of $\mathrm{Lp}(\mathrm{a})$. Besides numerous studies describing an elevation of $\operatorname{Lp}(\mathrm{a})$ in patients with end-stage kidney disease (reviewed in Kronenberg et $\mathrm{al}^{6}$ ), one study reported a large arteriovenous renovascular difference in $\mathrm{Lp}(\mathrm{a})$ plasma concentrations with lower values in the renal vein. $^{22}$ The latter study is in line with reports showing apo(a) fragments of various size in urine. ${ }^{23-26}$ The pathophysiological mechanism underlying the effect on Lp(a) levels, however, is unknown.

We described in two earlier studies (one included 534 hemodialysis patients) an apo(a) phenotype-associated elevation of $\operatorname{Lp}(a) .^{7,8}$ In our studies $\operatorname{Lp}(\mathrm{a})$ showed a relative increase only in hemodialysis patients with the so-called HMW apo(a) phenotypes when compared to phenotypematched controls, but not in those with LMW apo(a) phenotypes. This interesting but still unexplained phenomenon was confirmed by two recent reports ${ }^{9,10}$ and is supported by a phenotype-specific decrease of $\mathrm{Lp}(\mathrm{a})$ following kidney transplantation: in a prospective study including 154 patients, the average Lp(a) plasma concentration decreased during the first 3 weeks following transplantation only in the group of patients with HMW apo(a) phenotypes, but remained unchanged in those with LMW apo(a) types. ${ }^{14}$ In contrast to these observations, three smaller case-control studies observed an elevation of Lp(a) in hemodialysis patients in most of the apo(a) phenotype groups. ${ }^{11-13}$ Several reasons might be responsible for the discrepancy: ethnic differences between the populations investigated; different systems for the measurement of $\operatorname{Lp}(\mathrm{a})$ and apo(a) phenotyping as well as small sample sizes in the single phenotype subgroups. The inclusion of apo(a) isoforms with 23-25 K-IV repeats ( $\approx$ S3 according to the original nomenclature ${ }^{1}$ ) to the LMW subgroup ${ }^{13}$ might have influenced the outcome too, 


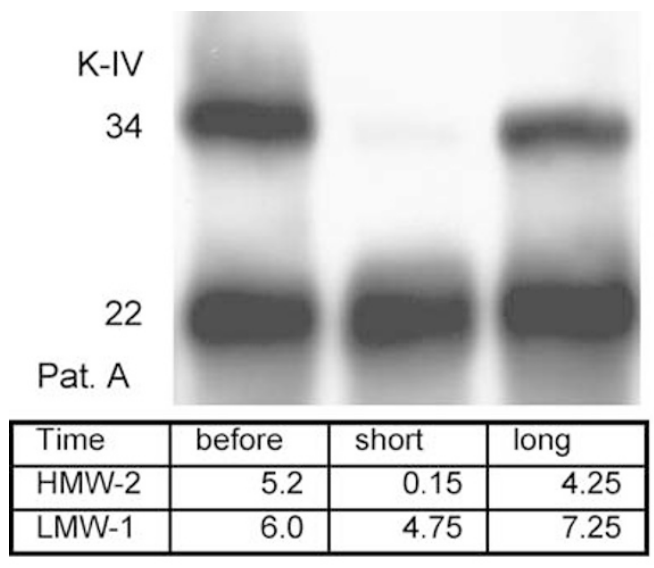

K-IV

28

18

Pat. B

\begin{tabular}{|l|r|r|}
\hline Time & \multicolumn{1}{|l|}{ before } & \multicolumn{1}{|c|}{ short } \\
\hline HMW-2 & 16 & 3 \\
\hline LMW-1 & 92 & 60 \\
\hline
\end{tabular}

K-IV

36

21

Pat. C

\begin{tabular}{|l|r|r|}
\hline Time & \multicolumn{1}{|c|}{ before } & \multicolumn{1}{|c|}{ short } \\
\hline HMW-2 & 6.4 & 3.8 \\
\hline LMW-1 & 2.8 & 4.3 \\
\hline
\end{tabular}
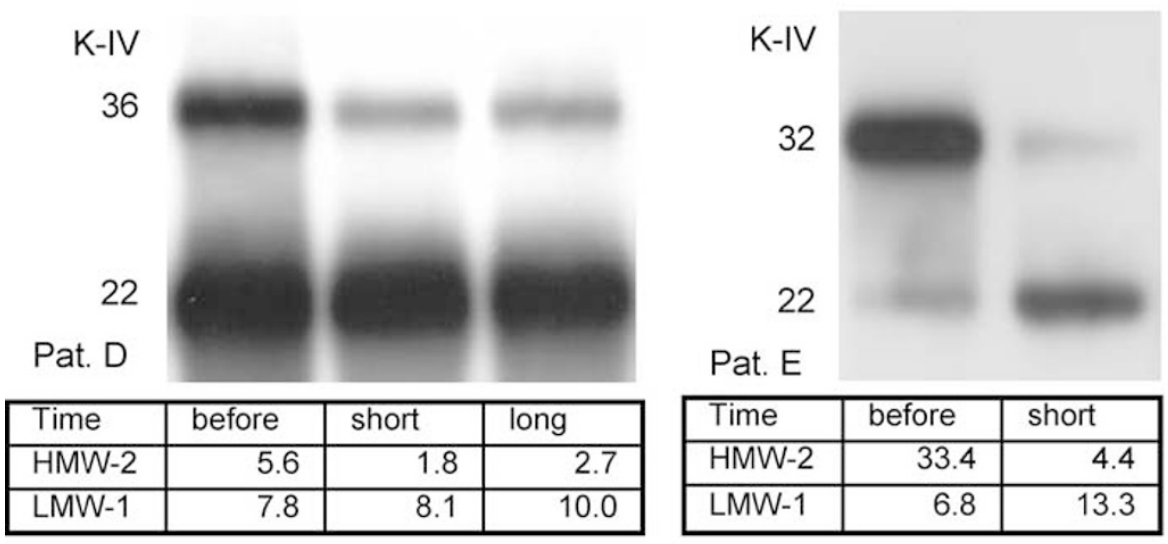

K-IV

35

29

Pat. F

\begin{tabular}{|l|r|r|}
\hline Time & \multicolumn{1}{|c|}{ before } & \multicolumn{1}{|c|}{ short } \\
\hline HMW-2 & 5.0 & 0.9 \\
\hline HMW-1 & 6.1 & 8.2 \\
\hline
\end{tabular}

K-IV

K-IV

35

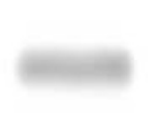

23

Pat. $\mathrm{H}$

Pat. G

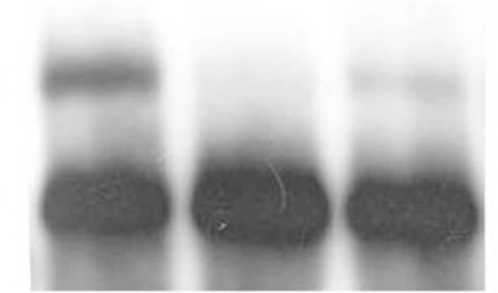

\begin{tabular}{|l|r|r|r|}
\hline Time & \multicolumn{1}{|l|}{ before } & \multicolumn{1}{l|}{ short } & \multicolumn{1}{l|}{ long } \\
\hline HMW-2 & 1.4 & 0.1 & 2.9 \\
\hline HMW-1 & 4.7 & 3.9 & 4.4 \\
\hline
\end{tabular}

Figure 3 Immunoblots of SDS agarose gel electrophoresis from eight representative patients showing the changes in the expression pattern of apo(a) isoforms after kidney transplantation. Plasma samples were withdrawn before transplantation and after a short-term (on average 3 weeks) and a long-term (on average 57 months) observation period following kidney transplantation. Whenever possible, we applied the same amount of apo(a) from the samples before and after transplantation from a given patient. We used the $L p(a)$ concentrations measured by ELISA to calculate the isoform-specific amount of $\operatorname{Lp}(a)$ derived from each isoform by using the densitometric relative estimates of the apo(a) bands (see Methods). 


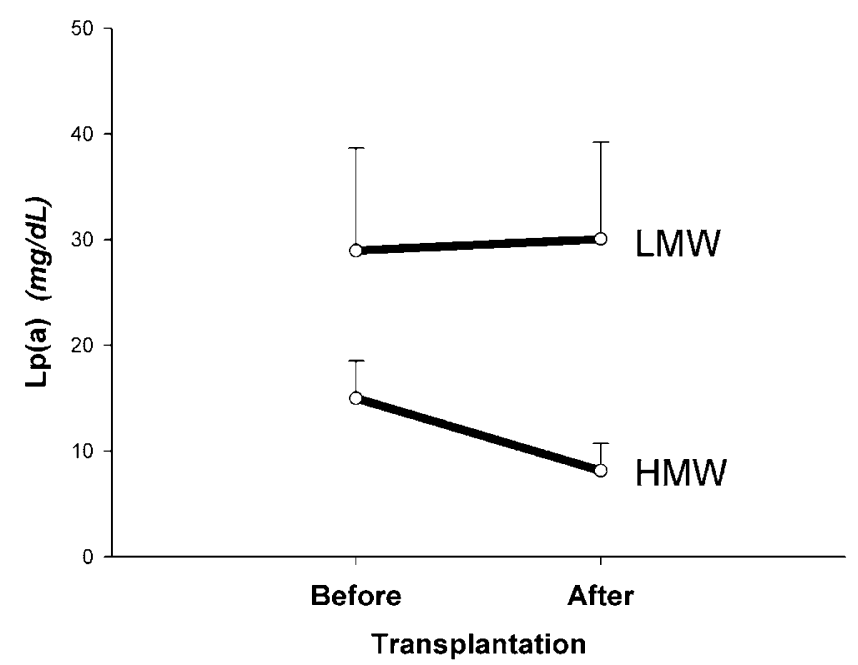

Figure 4 Interaction of kidney disease (kidney function) and apo(a) gene on apo(a) isoform-specific $\mathrm{Lp}(\mathrm{a})$ concentration changes. The effect of kidney disease is demonstrated by the recovery of kidney function by kidney transplantation. The apo(a) gene is represented either as low molecular weight (LMW) or high molecular weight (HMW) apo(a) isoform. Kidney disease has an effect on $\mathrm{Lp}(\mathrm{a})$ levels only in case of HMW apo(a) isoforms.

since we observed that this subgroup of patients has already a pronounced elevation of $\operatorname{Lp}(\mathrm{a}){ }^{7,8}$ In the present study, the relative decrease of $\operatorname{Lp}(\mathrm{a})$ was different for the various groups of HMW apo(a) isoforms: the combined results of both HMW isoforms in the HMW phenotype group showed a decrease of $\operatorname{Lp}(\mathrm{a})$ to $68 \%$ from the value before transplantation, which was less pronounced than in the group of HMW isoforms in the LMW phenotype group (decrease to $22 \%$ ). However, when we consider the absolute differences for $\mathrm{Lp}(\mathrm{a})$ levels between before and after kidney transplantation, we can observe a difference of $10.4 \mathrm{mg} / \mathrm{dl}$ for the HMW apo(a) isoform in patients with LMW apo(a) phenotypes. This was very similar to the total difference of $10.2 \mathrm{mg} / \mathrm{dl}$ for both HMW apo(a) isoforms in the HMW apo(a) phenotype group. It might be speculated that the absolute decrease of each single HMW apo(a) isoform, is smaller in the presence of two HMW apo(a) isoforms, since the full potential of an $\mathrm{Lp}(\mathrm{a})$-lowering effect is taped by two instead of one HMW isoform. Taken together, these data clearly show that the circulating levels of HMW apo(a) isoforms are significantly modulated by kidney function. One could speculate that the intact kidney clears Lp(a) of HMW isoforms from the circulation.

The study design used in the present study has major advantages for the investigation of an apo(a) genotype-dependent effect on $\operatorname{Lp}(\mathrm{a})$ in dialysis patients: first, it investigated prospectively the changes of the expression of apo(a) alleles during the phase of dialysis and following transplantation in each given individual. In other words, each patient is in her/his own control. Second, densitometric scanning of the apo(a) isoforms allows the calculation of the isoform-associated $\operatorname{Lp}(\mathrm{a})$ concentrations and therefore the specific changes of $\mathrm{Lp}(\mathrm{a})$ for each apo(a) allele. This procedure clearly demonstrated that in individuals expressing both an LMW and an HMW apo(a) isoform, a potential decrease of $\mathrm{Lp}(\mathrm{a})$ after transplantation is mainly caused by a decrease of $\operatorname{Lp}(\mathrm{a})$ particles containing an HMW apo(a) isoform. Third, changes in the relative expression of the lower to the higher molecular weight apo(a) isoforms before and following transplantation also indicate a different influence of renal insufficiency on Lp(a) particles with different apo(a) isoforms. This is most impressively demonstrated in patients in whom the HMW apo(a) band almost completely disappeared after transplantation (see Figure 3, patients A, B, F, G and $\mathrm{H}$ ) or in patients in whom the relative expression of the two alleles was reversed after transplantation (see Figure 3, patients $\mathrm{C}$ and $\mathrm{E}$ ).

We are aware of the apo(a) isoform-dependence of $\operatorname{Lp}(\mathrm{a})$ quantification procedures by ELISA and densitometric scanning since the used monoclonal anti-apo(a) antibody $1 \mathrm{~A} 2$ recognizes an epitope on the repetitive K-IV type-2 repeat of the apo(a) molecule. ${ }^{19}$ Our quantification could therefore eventually lead to overestimations of $\operatorname{Lp}(\mathrm{a})$ concentrations of HMW apo(a) isoforms. Owing to the longitudinal study design with a paired comparison of samples before and after transplantation, the main outcome and statement of this study will, nevertheless not be influenced by this phenomenon. It would rather have only an influence on the absolute $\operatorname{Lp}(\mathrm{a})$ levels than on the relative changes of $\operatorname{Lp}(\mathrm{a})$ and would therefore not influence the described interaction (Figure 4).

The analysis methods in the present study in any case minimize the problem of earlier studies, which categorized patients into groups of apo(a) phenotypes depending on the smaller apo(a) isoform or on the isoform that was expressed more dominantly. It explains also the observation in some patients expressing an LMW apo(a) isoform who nevertheless show a decrease of $\mathrm{Lp}(\mathrm{a})$ following kidney transplantation. In many cases, this decrease of Lp(a) might be caused by the often relatively large proportion of $\mathrm{Lp}(\mathrm{a})$ derived from the second expressed isoform, which in most cases is of HMW.

It has also to be stressed that this apo(a) isoform-specific decrease of $\operatorname{Lp}(\mathrm{a})$ after kidney transplantation is limited to patients treated by hemodialysis. Patients treated by CAPD show a decrease independent of the apo(a) isoforms as demonstrated recently ${ }^{27}$ and as observed impressively in one of our CAPD patients not presented in this report. The Lp(a) concentration of this patient dropped by $70 \mathrm{mg} / \mathrm{dl}$ (corresponding to about 50\%), which was caused by an equal decrease of both, the LMW and HMW apo(a) isoform. 


\section{Conclusions}

Our study clearly demonstrates that the changes of $\mathrm{Lp}(\mathrm{a})$ plasma concentrations in kidney disease depend on the genetically determined size of apo(a). This provides evidence for an interaction of apo(a) genetic variability and end-stage kidney disease on $\mathrm{Lp}(\mathrm{a})$ concentrations.

\section{Acknowledgements}

This study was supported by grants from the Austrian National Bank (Project 9331), the 'Österreichischer Herzfonds', the 'Else KrönerFresenius Stiftung' and the Knoll William Harvey Prize to Florian Kronenberg and by Grants P-12358 and P-15480 from the Austrian Science Fund to Hans Dieplinger and Gerd Utermann, respectively.

\section{References}

1 Utermann G, Menzel HJ, Kraft HG, Duba HC, Kemmler HG, Seitz C: $L p(a)$ glycoprotein phenotypes: inheritance and relation to Lp(a)-lipoprotein concentrations in plasma. J Clin Invest 1987; 80: 458-465.

2 Lackner C, Boerwinkle E, Leffert CC, Rahmig T, Hobbs HH: Molecular basis of apolipoprotein (a) isoform size heterogeneity as revealed by pulsed-field gel electrophoresis. J Clin Invest 1991; 87: 2153-2161.

3 Boerwinkle E, Leffert CC, Lin J, Lackner C, Chiesa G, Hobbs HH: Apolipoprotein(a) gene accounts for greater than $90 \%$ of the variation in plasma lipoprotein(a) concentrations. J Clin Invest 1992; 90: 52-60.

4 Kraft HG, Köchl S, Menzel HJ, Sandholzer C, Utermann G: The apolipoprotein(a) gene: a transcribed hypervariable locus controlling plasma lipoprotein(a) concentration. Hum Genet 1992; 90: 220-230.

5 Kronenberg F, Steinmetz A, Kostner GM, Dieplinger H: Lipoprotein(a) in health and disease. Crit Rev Clin Lab Sci 1996; 33: 495-543.

6 Kronenberg F, Utermann G, Dieplinger H: Lipoprotein(a) in renal disease. Am J Kidney Dis 1996; 27: 1-25.

7 Dieplinger H, Lackner C, Kronenberg F et al: Elevated plasma concentrations of lipoprotein(a) in patients with end-stage renal disease are not related to the size polymorphism of apolipoprotein(a). J Clin Invest 1993; 91: 397-401.

8 Kronenberg F, König P, Neyer U et al: Multicenter study of lipoprotein(a) and apolipoprotein(a) phenotypes in patients with end-stage renal disease treated by hemodialysis or continuous ambulatory peritoneal dialysis. J Am Soc Nephrol 1995; 6: 110-120.

9 Zimmermann J, Herrlinger S, Pruy A, Metzger T, Wanner C: Inflammation enhances cardiovascular risk and mortality in hemodialysis patients. Kidney Int 1999; 55: 648-658.

10 Milionis HJ, Elisaf MS, Tselepis A, Bairaktari E, Karabina SA, Siamopoulos KC: Apolipoprotein(a) phenotypes and lipoprotein(a) concentrations in patients with renal failure. Am J Kidney Dis 1999; 33: $1100-1106$

11 Hirata K, Kikuchi S, Saku K et al: Apolipoprotein(a) phenotypes and serum lipoprotein(a) levels in maintenance hemodialysis patients with/without diabetes mellitus. Kidney Int 1993; 44: 1062-1070.
12 Auguet T, Sentí M, Rubies-Prat J et al: Serum lipoprotein(a) concentration in patients with chronic renal failure receiving haemodialysis: influence of apolipoprotein(a) genetic polymorphism. Nephrol Dial Transplant 1993; 8: 1099-1103.

13 Gazzaruso C, Bonetti G, Garzaniti A et al: Increased plasma concentrations of lipoprotein(a) for every phenotype of apolipoprotein(a) in patients with chronic renal failure treated by hemodialysis. Nutr Metab Cardiovasc Dis 1996; 6: 203-210.

14 Kronenberg F, König P, Lhotta K et al: Apolipoprotein(a) phenotype-associated decrease in lipoprotein(a) plasma concentrations after renal transplantation. Arterioscler Thromb 1994; 14: 1399-1404.

15 Kronenberg F, Lobentanz E-M, König P, Utermann G, Dieplinger $\mathrm{H}$ : Effect of sample storage on the measurement of lipoprotein(a), apolipoproteins B and A-IV, total and high-density lipoprotein cholesterol and triglycerides. J Lipid Res 1994; 35: 1318-1328.

16 Kronenberg F, Trenkwalder E, Kronenberg MF, König P, Utermann $\mathrm{G}$, Dieplinger H: Influence of hematocrit on the measurement of lipoproteins demonstrated by the example of lipoprotein(a). Kidney Int 1998; 54: 1385-1389.

17 Kamboh MI, Ferrell RE, Kottke BA: Expressed hypervariable polymorphism of apolipoprotein (a). Am J Hum Genet 1991; 49: 1063-1074.

18 Kraft HG, Lingenhel A, Bader G, Kostner GM, Utermann G: The relative electrophoretic mobility of apo(a) isoforms depends on the gel system: proposal of a nomenclature for apo(a) phenotypes. Atherosclerosis 1996; 125: 53-61.

19 Dieplinger H, Gruber G, Krasznai K et al: Kringle 4 of human apolipoprotein[a] shares a linear antigenic site with human catalase. J Lipid Res 1995; 36: 813-822.

20 Kronenberg F, Kuen E, Ritz E et al: Lipoprotein(a) serum concentrations and apolipoprotein(a) phenotypes in mild and moderate renal failure. J Am Soc Nephrol 2000; 11: 105-115.

21 Koch M, Kutkuhn B, Trenkwalder E et al: Apolipoprotein B, fibrinogen, HDL cholesterol and apolipoprotein(a) phenotypes predict coronary artery disease in hemodialysis patients. J Am Soc Nephrol 1997; 8: 1889-1898.

22 Kronenberg F, Trenkwalder E, Lingenhel A et al: Renovascular arteriovenous differences in $\mathrm{Lp}(\mathrm{a})$ plasma concentrations suggest removal of $L p(a)$ from the renal circulation. J Lipid Res 1997; 38: $1755-1763$

23 Oida $\mathrm{K}$, Takai $\mathrm{H}$, Maeda $\mathrm{H}$ et al: Apolipoprotein(a) is present in urine and its excretion is decreased in patients with renal failure. Clin Chem 1992; 38: 2244-2248.

24 Mooser V, Seabra MC, Abedin M, Landschulz KT, Marcovina S, Hobbs HH: Apolipoprotein(a) kringle 4-containing fragments in urine. Relationship to plasma levels of lipoprotein(a). J Clin Invest 1996; 97: 858-864.

25 Kostner KM, Maurer G, Huber K et al: Urinary excretion of apo(a) fragments. Role in apo(a) catabolism. Arterioscler Thromb Vase Biol 1996; 16: 905-911.

26 Mooser V, Marcovina SM, White AL, Hobbs HH: Kringlecontaining fragments of apolipoprotein(a) circulate in human plasma and are excreted into the urine. J Clin Invest 1996; 98: 2414-2424.

27 Kerschdorfer L, König P, Neyer U et al: Lipoprotein(a) plasma concentrations after renal transplantation: a prospective evaluation after 4 years of follow-up. Atherosclerosis 1999; 144: 381-391. 